\title{
On the property between theta functions and Dedekind's function according to the changes in the value periods
}

\author{
Ismet Yildiz, Pinar Kahraman, Deniz Yetkin and Barbaros Celik
}

Department of Mathematics, Faculty of sciences and arts, Duzce University, Duzce, Turkey

Received: 3 April 2017, Accepted: 28 April 2017

Published online: 16 July 2017.

Abstract: In the study, two relations between the Dedekind's $\eta$-function and $\theta$-function were established by using characteristic values $\left(\varepsilon, \varepsilon^{\prime}\right) \equiv(0,0),(0,1)(\bmod 2)$ for $\theta$-function according to $\frac{1}{8}$ coefficient of period $\operatorname{pair}(u, \tau)$. The transformations among the theta functions according to the periods have been given and a Jacobian style elliptic functions has been set up the theta function by the help of a defined function.

Keywords: Theta functions,Dedekinds function, periods pair, charasteristics.

\section{Introduction}

It is of advantage to introduce function, defined by $\theta(\mathrm{u}, \tau)$, which has a rapidly convergent expansion in infinite series, and which is directly connected whith the sigma function of Weierstrass.Let $\tau$ be a complex variable, with $\tau=\frac{\omega_{2}}{\omega_{1}} \neq$ real, $\operatorname{Im} \tau>0$, and $\omega=\mathrm{m} \omega_{1}+\mathrm{n} \omega_{2}$ with $(\mathrm{m}, \mathrm{n}) \neq(0,0), m, n$ are integers and $u$ is a complex variable. We define the function $\theta(u, \tau)$ by the series,

$$
\theta\left[\begin{array}{c}
\varepsilon \\
\varepsilon^{\prime}
\end{array}\right](u, \tau)=\sum_{n} \exp \left\{\left(n+\frac{\varepsilon}{2}\right)^{2} \pi i \tau+2 \pi i\left(n+\frac{\varepsilon}{2}\right)\left(u+\frac{\varepsilon^{\prime}}{2}\right)\right\}
$$

where $\left(\varepsilon, \varepsilon^{\prime}\right)$ is $\left[\begin{array}{c}\varepsilon \\ \varepsilon^{\prime}\end{array}\right]$ and $\left[\begin{array}{c}\varepsilon \\ \varepsilon^{\prime}\end{array}\right] \equiv\left[\begin{array}{l}1 \\ 1\end{array}\right],\left[\begin{array}{l}0 \\ 1\end{array}\right],\left[\begin{array}{l}1 \\ 0\end{array}\right],\left[\begin{array}{l}0 \\ 0\end{array}\right](\bmod 2), \varepsilon$ and $\varepsilon^{\prime}$ are integers, and n ranges over all the integers $(-\infty$ to $\infty)$. The series in [1] converges absolutely, and uniformly in compact sets of the u-complex plane, and therefore represents an entire function of $\mathrm{u}[2]$. We can see the following alternative theta functions.

$$
\theta\left[\begin{array}{c}
\varepsilon \\
\varepsilon^{\prime}
\end{array}\right]\left(u+\frac{1}{2^{r}}+\frac{\tau}{2^{r}}, \tau\right)=\mu \theta\left[\begin{array}{c}
\varepsilon+\frac{1}{2^{r-1}} \\
\varepsilon^{\prime}+\frac{1}{2^{r-1}}
\end{array}\right]
$$

where $\mu=\exp \left\{-\frac{1}{4^{r}}(\tau+2) \pi \mathrm{i}-\frac{1}{2^{r}}\left(2 \mathrm{u}+\varepsilon^{\prime}\right) \pi \mathrm{i}\right\}$ and $\left[\begin{array}{l}\varepsilon \\ \varepsilon^{\prime}\end{array}\right] \equiv\left[\begin{array}{l}1 \\ 1\end{array}\right],\left[\begin{array}{l}0 \\ 1\end{array}\right],\left[\begin{array}{l}1 \\ 0\end{array}\right],\left[\begin{array}{l}0 \\ 0\end{array}\right](\bmod 2)$, and $\varepsilon^{\prime}$ are integers in [2].

$$
\theta\left[\begin{array}{c}
\varepsilon \\
\varepsilon^{\prime}
\end{array}\right](u, \tau)=\sum_{n} \exp \left\{\left(n+\frac{\varepsilon}{2}\right)^{2} \pi i \tau+2 \pi i\left(n+\frac{\varepsilon}{2}\right)\left(u+\frac{\varepsilon^{\prime}}{2}\right)\right\}
$$


where $\left[\begin{array}{c}\varepsilon \\ \varepsilon^{\prime}\end{array}\right] \equiv\left[\begin{array}{l}1 \\ 1\end{array}\right],\left[\begin{array}{l}0 \\ 1\end{array}\right],\left[\begin{array}{l}1 \\ 0\end{array}\right],\left[\begin{array}{l}0 \\ 0\end{array}\right](\bmod 2), \varepsilon$ and $\varepsilon^{\prime}$ are integers $n$ ranges over all the integers $(-\infty$ to $\infty)$ in [2].

$$
\theta\left[\begin{array}{c}
\varepsilon \\
\varepsilon^{\prime}
\end{array}\right](u, \tau)=\sum_{n} \exp \left\{\left(n+\frac{\varepsilon}{2}\right)^{2} \pi i \tau+2 i\left(n+\frac{\varepsilon}{2}\right)\left(u-\frac{\varepsilon^{\prime}}{2} \pi\right)\right\}
$$

where $\left[\begin{array}{c}\varepsilon \\ \varepsilon^{\prime}\end{array}\right] \equiv\left[\begin{array}{l}1 \\ 1\end{array}\right],\left[\begin{array}{l}0 \\ 1\end{array}\right],\left[\begin{array}{l}1 \\ 0\end{array}\right],\left[\begin{array}{l}0 \\ 0\end{array}\right](\bmod 2), \varepsilon$ and $\varepsilon^{\prime}$ are integers $n$ ranges over all the integers $(-\infty$ to $\infty)$ in [4]. If $\left[\begin{array}{c}\varepsilon \\ \varepsilon^{\prime}\end{array}\right] \equiv\left[\begin{array}{l}1 \\ 1\end{array}\right](\bmod 2)$ then $\theta\left[\begin{array}{l}1 \\ 1\end{array}\right](u, \tau)=-i \sum_{n}(-1)^{n} \exp \left\{\left(n+\frac{1}{2}\right)^{2} \pi i \tau+(2 n+1) \pi i u\right\}$ This function $\theta\left[\begin{array}{l}1 \\ 1\end{array}\right](u, \tau)$ is an alternative formula in [4]. in $[4]$.

If $\left[\begin{array}{c}\varepsilon \\ \varepsilon^{\prime}\end{array}\right] \equiv\left[\begin{array}{l}0 \\ 0\end{array}\right](\bmod 2)$ and $u=0$ then the function $\theta\left[\begin{array}{l}1 \\ 1\end{array}\right](u, \tau)$ is an alternative formula in [4].

If $\left[\begin{array}{c}\varepsilon \\ \varepsilon^{\prime}\end{array}\right] \equiv\left[\begin{array}{l}0 \\ 0\end{array}\right](\bmod 2)$ and $\mathrm{u}=0$ then $\theta\left[\begin{array}{l}0 \\ 0\end{array}\right](0, \tau)=\sum_{n} \exp \left(n^{2} \pi i \tau\right)$ The function $\theta\left[\begin{array}{l}0 \\ 0\end{array}\right](0, \tau)$ is an alternative formula

Definition 1. A period, denoted $\left\{\begin{array}{l}a \\ b\end{array}\right\}$, is $b+a \tau$.

$$
\text { if } r=3 \text { then } \frac{1}{8}\left\{\begin{array}{l}
a \\
b
\end{array}\right\}=\frac{b}{8}+\frac{a \tau}{8} \text {. }
$$

A reduced quarter-period is a quarter-period in which a,b equal 0 or 1 [4]. With the help of this alternativa formula in [2]. Above, we can get the following equalities according to quarter-periods. If $\left[\begin{array}{c}\varepsilon \\ \varepsilon^{\prime}\end{array}\right] \equiv\left[\begin{array}{l}1 \\ 1\end{array}\right](\bmod 2)$ then

$$
\begin{aligned}
\theta\left[\begin{array}{l}
1 \\
1
\end{array}\right]\left(u+\frac{1}{8}\left\{\begin{array}{l}
1 \\
1
\end{array}\right\}, \tau\right) & =\sum_{n} \exp \left\{\left(n+\frac{1}{2}\right)^{2} \pi i \tau+2 \pi i\left(n+\frac{1}{2}\right)\left(u+\frac{1}{8}\left\{\begin{array}{l}
1 \\
1
\end{array}\right\}+\frac{1}{4}\right\}\right. \\
& =i e^{-\frac{\pi i \tau}{4}} \sum_{n}(-1)^{n} \exp \left\{\left(n+\frac{1}{2}\right)^{2} \pi i \tau+(2 n+1) \pi i u+\frac{n \pi i}{2}+\frac{n \pi i \tau}{2}+\frac{\pi i \tau}{4}+\frac{\pi i}{4}\right\}
\end{aligned}
$$

If $\left[\begin{array}{c}\varepsilon \\ \varepsilon^{\prime}\end{array}\right] \equiv\left[\begin{array}{l}1 \\ 0\end{array}\right](\bmod 2)$ then

$$
\begin{aligned}
\theta\left[\begin{array}{l}
1 \\
0
\end{array}\right]\left(u+\frac{1}{8}\left\{\begin{array}{l}
1 \\
1
\end{array}\right\}, \tau\right) & =\sum_{n} \exp \left\{\left(n+\frac{\varepsilon}{2}\right)^{2} \pi i \tau+2 \pi i\left(n+\frac{1}{2}\right)\left(u+\frac{1}{8}\left\{\begin{array}{l}
1 \\
1
\end{array}\right\}\right\}\right. \\
& =e^{-\frac{\pi i \tau}{4}} \sum_{n} \exp \left\{\left(n+\frac{1}{2}\right)^{2} \pi i \tau+(2 n+1) \pi i u+\frac{n \pi i}{4}+\frac{n \pi i \tau}{4}+\frac{\pi i \tau}{8}+\frac{\pi i}{8}\right\}
\end{aligned}
$$

Using the equations we can get

$$
\frac{\theta\left[\begin{array}{l}
1 \\
1
\end{array}\right]\left(u+\frac{1}{8}\left\{\begin{array}{l}
1 \\
1
\end{array}\right\}, \tau\right)}{\theta\left[\begin{array}{l}
1 \\
0
\end{array}\right]\left(u+\frac{1}{8}\left\{\begin{array}{l}
1 \\
1
\end{array}\right\}, \tau\right)}=\frac{i e^{-\frac{\pi i \tau}{8}} \sum_{n}(-1)^{n} \exp \left\{\left(n+\frac{1}{2}\right)^{2} \pi i \tau+(2 n+1) \pi i u+\frac{n \pi i}{4}+\frac{n \pi i \tau}{4}+\frac{\pi i \tau}{8}+\frac{\pi i}{8}\right\}}{e^{-\frac{\pi i \tau}{8}} \sum_{n} \exp \left\{\left(n+\frac{1}{2}\right)^{2} \pi i \tau+(2 n+1) \pi i u+\frac{n \pi i}{4}+\frac{n \pi i \tau}{4}+\frac{\pi i \tau}{8}+\frac{\pi i}{8}\right\}}
$$


(i) If $n$ is 0 or even integer then, $\theta\left[\begin{array}{l}1 \\ 1\end{array}\right]\left(u+\frac{1}{8}\left\{\begin{array}{l}1 \\ 1\end{array}\right\}, \tau\right)=i \theta\left[\begin{array}{l}1 \\ 0\end{array}\right]\left(u+\frac{1}{8}\left\{\begin{array}{l}1 \\ 1\end{array}\right\}, \tau\right)$.

(ii) If $n$ is odd integer then $\theta\left[\begin{array}{l}1 \\ 1\end{array}\right]\left(u+\frac{1}{8}\left\{\begin{array}{l}1 \\ 1\end{array}\right\}, \tau\right)=-i \theta\left[\begin{array}{l}1 \\ 0\end{array}\right]\left(u+\frac{1}{8}\left\{\begin{array}{l}1 \\ 1\end{array}\right\}, \tau\right)$.

If $\left[\begin{array}{c}\varepsilon \\ \varepsilon^{\prime}\end{array}\right] \equiv\left[\begin{array}{l}0 \\ 1\end{array}\right](\bmod 2)$ then

$$
\begin{aligned}
\theta\left[\begin{array}{l}
0 \\
1
\end{array}\right]\left(u+\frac{1}{8}\left\{\begin{array}{l}
1 \\
1
\end{array}\right\}, \tau\right) & =\sum_{n} \exp \left\{n^{2} \pi i \tau+2 n \pi i\left(u+\frac{1}{8}\left\{\begin{array}{l}
1 \\
1
\end{array}\right\}+\frac{1}{4}\right\}\right. \\
& =\sum_{n}(-1)^{n} \exp \left\{n^{2} \pi i \tau+2 n \pi i u+\frac{n \pi i}{4}+\frac{n \pi i \tau}{4}\right\}
\end{aligned}
$$

If $\left[\begin{array}{c}\varepsilon \\ \varepsilon^{\prime}\end{array}\right] \equiv\left[\begin{array}{l}0 \\ 0\end{array}\right](\bmod 2)$ then

$$
\begin{aligned}
\theta\left[\begin{array}{l}
0 \\
0
\end{array}\right]\left(u+\frac{1}{8}\left\{\begin{array}{l}
1 \\
1
\end{array}\right\}, \tau\right) & =\sum_{n} \exp \left\{n^{2} \pi i \tau+2 n \pi i\left(u+\frac{1}{8}\left\{\begin{array}{l}
1 \\
1
\end{array}\right\}\right\}\right. \\
& =\sum_{n} \exp \left\{n^{2} \pi i \tau+2 n \pi i u+\frac{n \pi i}{4}+\frac{n \pi i \tau}{4}\right\}
\end{aligned}
$$

From the above equations we obtain

$$
\frac{\theta\left[\begin{array}{l}
0 \\
1
\end{array}\right]\left(u+\frac{1}{8}\left\{\begin{array}{l}
1 \\
1
\end{array}\right\}, \tau\right)}{\theta\left[\begin{array}{l}
0 \\
0
\end{array}\right]\left(u+\frac{1}{8}\left\{\begin{array}{l}
1 \\
1
\end{array}\right\}, \tau\right)}=\frac{\sum_{n}(-1)^{n} \exp \left\{n^{2} \pi i \tau+2 n \pi i u+\frac{n \pi i}{4}+\frac{n \pi i \tau}{4}\right\}}{\sum_{n} \exp \left\{n^{2} \pi i \tau\left(2 n \pi i u+\frac{n \pi i}{4}+\frac{n \pi i \tau}{4}\right\}\right.}
$$

(iii) If $n$ is 0 or even integer then

$$
\theta\left[\begin{array}{l}
0 \\
1
\end{array}\right]\left(u+\frac{1}{8}\left\{\begin{array}{l}
1 \\
1
\end{array}\right\}, \tau\right)=\theta\left[\begin{array}{l}
0 \\
0
\end{array}\right]\left(u+\frac{1}{8}\left\{\begin{array}{l}
1 \\
1
\end{array}\right\}, \tau\right)
$$

(iv) If $\mathrm{n}$ is odd integer then

$$
\theta\left[\begin{array}{l}
0 \\
1
\end{array}\right]\left(u+\frac{1}{8}\left\{\begin{array}{l}
1 \\
1
\end{array}\right\}, \tau\right)=\theta\left[\begin{array}{l}
0 \\
0
\end{array}\right]\left(u+\frac{1}{8}\left\{\begin{array}{l}
1 \\
1
\end{array}\right\}, \tau\right)
$$

Theorem 1. The function $\theta\left[\begin{array}{l}1 \\ 1\end{array}\right](u, \tau)$ defined in [3] is odd function of $u$ and it can be expressed by infinite product

$$
\theta\left[\begin{array}{l}
1 \\
1
\end{array}\right](u, \tau)=c e^{\frac{\pi i \tau}{4}} 2 \sin \pi u \prod_{n=1}^{\infty}\left\{1-e^{2(n \tau+u) \pi i}\right\} \prod_{n=1}^{\infty}\left\{1-e^{2(n \tau-u) \pi i}\right\}
$$

where $c=\prod_{n=1}^{\infty}\left(1-e^{2 n \pi i \tau}\right)$, Imt $>0[2]$. We consider the function $\phi(u, \tau)$ expressed by product

$$
\varphi(u, \tau)=\prod_{n=1}^{\infty}\left\{1-e^{[(2 n-1) \tau+2 u] \pi i}\right\} \prod_{n=1}^{\infty}\left\{1-e^{[(2 n-1) \tau-2 u] \pi i}\right\} .
$$


Theorem 2. The function $\frac{\theta\left[\begin{array}{l}1 \\ 1\end{array}\right](u, \tau)}{\varphi(u, \tau)}$ is a elliptic function with periods 1 and $\tau$.

Proof. Let $\psi(u, \tau)=\frac{\theta\left[\begin{array}{l}1 \\ 1\end{array}\right](u, \tau)}{\varphi(u, \tau)}$, then we write

$$
\psi(u+1, \tau)=\frac{\theta\left[\begin{array}{l}
1 \\
1
\end{array}\right](u+1, \tau)}{\varphi(u+1, \tau)}=\frac{-\theta\left[\begin{array}{l}
1 \\
1
\end{array}\right](u, \tau)}{\varphi(u, \tau)}=\frac{\theta\left[\begin{array}{l}
1 \\
1
\end{array}\right](-u, \tau)}{\varphi(u, \tau)}
$$

where $\theta\left[\begin{array}{l}1 \\ 1\end{array}\right](u, \tau)=-\theta\left[\begin{array}{l}1 \\ 1\end{array}\right](-u, \tau)$ from theorem 2 .

$$
\psi(u+\tau, \tau)=\frac{\theta\left[\begin{array}{l}
1 \\
1
\end{array}\right](u+\tau, \tau)}{\varphi(u+\tau, \tau)}=\frac{-e^{-(2 u+\tau) \pi i} \theta\left[\begin{array}{l}
1 \\
1
\end{array}\right](u, \tau)}{e^{-(2 u+\tau) \pi i} \varphi(u, \tau)}=\frac{\theta\left[\begin{array}{l}
1 \\
1
\end{array}\right](u, \tau)}{\varphi(u, \tau)}
$$

since

$$
\begin{aligned}
\phi(u+\tau, \tau) & =\prod_{n=1}^{\infty}\left\{1-e^{(2 n-1) \tau \pi i+2 \pi i(u+\tau)}\right\} \prod_{n=1}^{\infty}\left\{1-e^{(2 n-1) \tau \pi i-2 \pi i(u+\tau)}\right\} \\
& =\prod_{n=1}^{\infty}\left\{1-e^{(2 n+1) \tau \pi i+2 \pi i u}\right\} \prod_{n=1}^{\infty}\left\{1-e^{(2 n-3) \tau \pi i-2 \pi i u}\right\} \\
& =\prod_{n=1}^{\infty}\left\{1-e^{[2(n+1)-1] \tau \pi i+2 \pi i u}\right\} \prod_{n=1}^{\infty}\left\{1-e^{[2(n-1)-1] \tau \pi i-2 \pi i u}\right\} \\
& =\prod_{m=2}^{\infty}\left\{1-e^{(2 n+1) \tau \pi i+2 \pi i u}\right\} \prod_{n=0}^{\infty}\left\{1-e^{((2 n-3) \tau \tau i-2 \tau u i}\right\} \\
& =\prod_{m=1}^{\infty}\left\{1-e^{(2 n+1) \tau \pi i+2 \pi i u}\right\} \prod_{m=1}^{\infty}\left\{1-e^{(2 m-1) \tau \pi i-2 \pi i u}\right\}\left\{1-e^{-(\pi i \tau+2 \pi i u)}\right\}\left\{1-e^{(\pi i \tau+2 \pi i u)}\right\}^{-1} \\
& =-e^{-(2 u+\tau) \pi i} \phi(u, \tau)
\end{aligned}
$$

The function $\psi(u, \tau)$ is therefore a doubly periodic with periods 1 and $\tau$ having neither zeros nor poles on account of the fact that $\theta\left[\begin{array}{l}1 \\ 1\end{array}\right](u, \tau)$ possesses the same periodicity factors as $\phi(\mathrm{u}, \tau)$. Hence the function $\psi(u, \tau)$ is an elliptic function since the set of all meromorphic functions form a field and $\psi(u, \tau)$ is meromorphic and periodic with periods 1 and $\tau$. [2], [4].

We first define the Riemann's $\theta$-function by the series

$$
\theta\left[\begin{array}{l}
a \\
b
\end{array}\right](u, \tau)=\sum_{n=-\infty}^{\infty} \exp \left\{(n+a)^{2} \pi i \tau+2 \pi i(n+a)(u+b)\right\}
$$

with a given complex number $\mathrm{u}$, and complex number $\tau$ satisfying $\operatorname{Im}\left(\tau=\frac{\omega_{1}}{\omega_{2}} \neq\right.$ real $)>0$ and characteristic $\left[\begin{array}{l}a \\ b\end{array}\right]$ where $a, b$ are rational numbers. Let us recall the transformation $n^{2}+n=n(n+1)$ is congruent to zero module $2\left({ }^{2} \mid n(n+1)\right.$ where $n \equiv 0(\bmod 2)$ and $^{2} \mid n(n+1)$ from $n+1 \equiv 0(\bmod 2)$ if $\left.n \equiv 1(\bmod 2)\right)[4]$. 
In this article, we treat the special value of $\theta$ - function with characteristic $\left[\begin{array}{c}\varepsilon \\ \varepsilon^{\prime}\end{array}\right]$

$$
\theta\left[\begin{array}{c}
\varepsilon \\
\varepsilon^{\prime}
\end{array}\right](u, \tau)=\sum_{n=-\infty}^{\infty} \exp \left\{\left(n+\frac{\varepsilon}{2}\right)^{2} \pi i \tau+2 \pi i\left(n+\frac{\varepsilon}{2}\right)\left(u+\frac{\varepsilon^{\prime}}{2}\right)\right\}
$$

where $\left[\begin{array}{c}\varepsilon \\ \varepsilon^{\prime}\end{array}\right] \equiv\left[\begin{array}{l}0 \\ 1\end{array}\right],\left[\begin{array}{l}0 \\ 0\end{array}\right](\bmod 2)$,and $\varepsilon, \varepsilon^{\prime}$ are integers [6]. Thus, we have the following relations

$$
\theta\left[\begin{array}{l}
0 \\
1
\end{array}\right](u, \tau)=\sum_{n=-\infty}^{\infty}(-1)^{n} \exp \left(n^{2} \pi i \tau+2 n \pi i u\right)
$$

Now , let us observe that

$$
\theta\left[\begin{array}{l}
0 \\
0
\end{array}\right](u, \tau)=\sum_{n} \exp \left(n^{2} \pi i \tau+2 n \pi i u\right)
$$

Then we see

$$
\theta\left[\begin{array}{l}
0 \\
0
\end{array}\right](u, \tau)=\sum_{n=-\infty}^{\infty}\left(\exp \left(n^{2} \pi i \tau+2 n \pi i u\right)\right.
$$

that the function $\theta\left[\begin{array}{l}0 \\ 0\end{array}\right](0, \tau)$ defined by the series in [1] is a alternative formula of $\theta\left[\begin{array}{l}\varepsilon \\ \varepsilon^{\prime}\end{array}\right](u, \tau)$.The formulas $\theta\left[\begin{array}{l}0 \\ 0\end{array}\right](u, \tau)$ and $\theta\left[\begin{array}{l}0 \\ 1\end{array}\right](u, \tau)$ were used in this article where $u \neq 0$. At first we see the infinite products

$$
\theta\left[\begin{array}{l}
0 \\
0
\end{array}\right](u, \tau)=\prod_{n=1}^{\infty}\left(1-e^{2 n \pi i \tau}\right) \cdot \prod_{n=1}^{\infty}\left(1+e^{(2 n-1) \pi i \tau+2 \pi i u}\right) \cdot \prod_{n=1}^{\infty}\left(1+e^{(2 n-1) \pi i \tau-2 \pi i u}\right) .
$$

which it converges absolutely [2] .

Theorem 3. We have the relations

(i) $\eta(u)=e^{\frac{\pi i u}{12}} \cdot \theta\left[\begin{array}{l}0 \\ 0\end{array}\right]\left(\frac{u+6}{4}, \tau\right)$.

(ii) $\theta\left[\begin{array}{l}0 \\ 1\end{array}\right]\left(\frac{u+6}{4}, \tau\right)=e^{-\frac{\pi i u}{12}} \eta(u) . \prod_{n=1}^{\infty}\left(1-e^{(2 n-1) \pi i u}\right)$ between the functions $\theta\left[\begin{array}{l}0 \\ 0\end{array}\right](u, \tau), \theta\left[\begin{array}{l}0 \\ 1\end{array}\right](u, \tau)$ and Dedekind's $\eta$-function which defined by the infinite product

$$
\eta(u)=e^{\frac{\pi i u}{12}} \cdot \prod_{n=1}^{\infty}\left(1-e^{2 n \pi i u}\right)
$$

where $\operatorname{Im} \tau>0$ and $k$ is a integer [6].

Proof.

(i) Let us recall the formula

$$
\theta\left[\begin{array}{l}
0 \\
0
\end{array}\right](u, \tau)=\prod_{n=1}^{\infty}\left(1-e^{2 n \pi i \tau}\right) \cdot \prod_{n=1}^{\infty}\left(1+e^{(2 n-1) \pi i \tau+2 \pi i u}\right) \cdot \prod_{n=1}^{\infty}\left(1+e^{(2 n-1) \pi i \tau-2 \pi i u}\right) .
$$


If $\mathrm{k}$ integer, then we have

$$
\begin{aligned}
\theta\left[\begin{array}{l}
0 \\
0
\end{array}\right](3 u+2 k, \tau) & =\prod_{n=1}^{\infty}\left(1-e^{2 n \pi i(3 u+2 k)}\right) \cdot \prod_{n=1}^{\infty}\left(1+e^{(2 n-1) \pi i(3 u+2 k)+2 \pi i \tau}\right) \cdot \prod_{n=1}^{\infty}\left(1+e^{(2 n-1) \pi i(3 u+2 k)-2 \pi i \tau}\right) \\
& =\prod_{n=1}^{\infty}\left(1-e^{6 n \pi i u}\right) \cdot \prod_{n=1}^{\infty}\left(1+e^{6 n \pi i u-2 \pi i u-(2 k-1) \pi i}\right) \cdot \prod_{n=1}^{\infty}\left(1+e^{6 n \pi i u-4 \pi i u-(2 k+1) \pi i}\right) \\
& =\prod_{n=1}^{\infty}\left(1-e^{6 n \pi i u}\right) \cdot \prod_{n=1}^{\infty}\left(1-e^{6 n \pi i u-2 \pi i u}\right) \cdot \prod_{n=1}^{\infty}\left(1-e^{6 n \pi i u-4 \pi i u}\right)
\end{aligned}
$$

If we set $G=e^{2 \pi i u}$, then we obtain

$$
\theta\left[\begin{array}{l}
0 \\
0
\end{array}\right](3 u+2 k, \tau)=\prod_{n=1}^{\infty}\left(1-G^{3 n}\right) \cdot \prod_{n=1}^{\infty}\left(1-G^{3 n-1}\right) \cdot \prod_{n=1}^{\infty}\left(1-G^{3 n-2}\right) .
$$

On the other hand, we may set $n=m+1$, then

$$
\begin{aligned}
\theta\left[\begin{array}{l}
0 \\
0
\end{array}\right](3 u+2 k, \tau) & =\prod_{n^{\prime}=1}^{\infty}\left(1-G^{3 m+3}\right) \cdot \prod_{n^{\prime}=1}^{\infty}\left(1-G^{3 m+2}\right) \cdot \prod_{n^{\prime}=1}^{\infty}\left(1-G^{3 m+1}\right) \\
& =(1-G)\left(1-G^{2}\right)\left(1-G^{3}\right)\left(1-G^{4}\right)=\cdots \\
& =\prod_{m=1}^{\infty}\left(1-G^{m}\right)=\prod_{m=1}^{\infty}\left(1-e^{2 m \pi i u}\right) .
\end{aligned}
$$

According to above equations, we have

$$
\eta(u)=e^{\frac{\pi i u}{12}} \cdot \theta\left[\begin{array}{l}
0 \\
0
\end{array}\right](3 u+2 k, \tau)
$$

from the Dedekind's $\eta$-function defined by the infinite product

$$
\eta(u)=e^{\frac{\pi i u}{12}} \cdot \prod_{n=1}^{\infty}\left(1-e^{2 n \pi i u}\right)
$$

(ii) According to the equation,

$$
\theta\left[\begin{array}{l}
0 \\
1
\end{array}\right](u, \tau)=\sum_{n=-\infty}^{\infty}(-1)^{n} \exp \left(n^{2} \pi i \tau+2 n \pi i u\right)
$$

we have

$$
\begin{aligned}
\theta\left[\begin{array}{l}
0 \\
1
\end{array}\right]\left(\frac{u+6}{4}, \tau\right) & =\sum_{n=-\infty}^{\infty}(-1)^{n} \exp \left[\frac{1}{2} n(3 n+1) \pi i u\right] \\
& =1+\sum_{n=1}^{\infty}(-1)^{n}\left\{\exp \left[\frac{1}{2} n(3 n-1) \pi i u\right]+\exp \left[\frac{1}{2} n(3 n+1) \pi i u\right]\right\}
\end{aligned}
$$

Where $\frac{1}{2} n(3 n+1)$ are the pentagonal numbers and $\mathrm{n}$ is negative integers [4].

This results play a role of key stone in the forthcoming work concerning relation between the $\theta$-theta function and Dedekind's $\eta$-function. In fact, if the application of theorem.3(ii)on the relation obtained with the theorem.3.(i) which known as the equation between Dedekind's $\eta$-function and L.Euler's theorem on pentagonal numbers is 
done, we obtain

$$
\begin{aligned}
\frac{\theta\left[\begin{array}{l}
0 \\
1
\end{array}\right]\left(\frac{u+6}{4}, \tau\right)}{\prod_{n=1}^{\infty}\left(1-e^{(2 n-1) \pi i u)}\right.}=\frac{\sum_{n=-\infty}^{\infty}(-1)^{n} \exp \left(\frac{1}{2} n(3 n+1) \pi i u\right)}{\prod_{n=1}^{\infty}\left(1-e^{(2 n-1) \pi i u}\right)} \\
=\frac{\prod_{n=1}^{\infty}\left(1-e^{n \pi i u}\right)}{\prod_{n=1}^{\infty}\left(1-e^{(2 n-1) \pi i u}\right)}=\prod_{n=1}^{\infty}\left(1-e^{2 n \pi i u}\right)=e^{-\frac{\pi i u}{12}} \eta(u) .
\end{aligned}
$$

As a result, the relation has been obtained between theta and Dedkind's $-\eta(u)$ functions by using the characteristic $\left[\begin{array}{l}0 \\ 1\end{array}\right]$ and the variable $\frac{u+6}{4}$ instead of the characteristic $\left[\begin{array}{l}0 \\ 1\end{array}\right]$ and the variable $\frac{u+4}{4}$ which were previously used by [4].

\section{Conclusion}

A new correlation was established between theta and Dedekind eta functions using 1/8 times of periods and an appropriate value of the variable.

\section{Competing interests}

The authors declare that they have no competing interests.

\section{Authors' contributions}

All authors have contributed to all parts of the article. All authors read and approved the final manuscript.

\section{References}

[1] Gregory L. Wilson, A family of modular functions Arising from the theta function London Math. Soc. (3),55,1987, London.

[2] Harry E.Rauch, Elliptic Functions, Theta Functions, and Riemann surfaces, The Library of Congress catalog card number 7488141, SBN 683-07187-4, 1973 Baltimore, USA.

[3] Komaravolu, C., Elliptic functions, Springer-Verlag Berlin Heidelberg New York Tokyo, ISBN 0-387-15295-4,1985, Germany.

[4] Yıldız.I., Uyanık. N., "On The Elliptic Function Arising From the Theta Functions and Dedekind's -function" Journal of Math. and Stat.1 (2):153-159 ( 2005).

[5] Yildiz, I., On extension of the modular transformations over the modular group by reflection" journal of applied mathematics and computation. 153 (2004).111-116.

[6] Tom M. Apostol., Modular functions and Dirichled Series in Number theory.California institute of technology. January.1976. 